\section{Geschlechtsspezifische Ansätze in der Suchttherapie}

Zusammenfassung: Anliegen: Es werden verschiedene Faktoren aufgezeigt, die auf geschlechtsspezifische Unterschiede im Zusammenhang mit Suchterkrankungen hinweisen und deren nähere Untersuchung die Voraussetzung für die Etablierung geschlechtssensibler Therapieansätze liefern sollte. Ergebnisse: Substanzabhängige Frauen weisen wesentliche Unterschiede bezüglich Prävalenz, Komorbidität, Verlauf, Therapieansprechen und Morbidität in ihrer Erkrankung im Vergleich zu männlichen substanzabhängigen Patienten auf. Schlussfolgerungen: Da die geschlechtsspezifische Forschung in der Suchterkrankung langsam erste Fortschritte aufzeigt und durch neuere bildgebende Verfahren eine unterschiedliche Verstoffwechselung der Substanzen nachgewiesen wurde, sollten entsprechende Erkenntnisse auch im therapeutischen Bereich umgesetzt werden. Eine entsprechende Anzahl von weiblichen Probandinnen ist daher bei klinischen Studien zu fordern und die öffentliche Forschungsgeldvergabe sollte dies berücksichtigen.

Schlüsselwörter: Substanzgebrauchsstörungen - Geschlechtsspezifische Unterschiede - Geschlechtssensible Therapieansätze

Gender Related Approaches in Addiction Treatment: Objective: The aim of our study was to perform an analysis of different variables to determine the important factors of gender related substance disorders in order to define established gender sensitive therapies. Results: Substance dependent women show significant differences in prevalence, personality structure, comorbidity, therapy outcome and morbidity in comparison to the male target group. Conclusions: As the topic of gender specific issues in substance dependence is still underrepresented in psychiatric science, and under the consideration of new research results emphasizing the need of specific treatment approaches, further studies are needed to investigate this focus. Enhanced enrollment of women in clinical study design in combination with increased public funding might overcome the so far minority group.

Key words: Substance Use Disorders - Gender Related Differences - Gender Sensitive Treatment Programs

Suchttherapie Sonderheft 2001; 2: S6-S10

(c) Georg Thieme Verlag Stuttgart · New York ISSN 1439-9903
Anita Schreiberhuber, Werner Schlegel, Arif Mendelsohn, Gabriele Fischer

Drogenambulanz, Klinische Abteilung für Allgemeine Psychiatrie, Universitätsklinik für Psychiatrie, AKH Wien

\section{Einleitung}

Wenn auch die Erforschung geschlechtsspezifischer Unterschiede bei Suchterkrankungen zunehmend an Bedeutung gewinnt, stellt sich bei näherer Betrachtung der diesbezüglich vorhandenen Literatur heraus, dass die Mehrzahl der Probanden männlichen Geschlechts ist und nach wie vor sehr wenige Untersuchungen bezüglich Prävalenz, Verlauf und Therapie bei substanzabhängigen Frauen vorliegen [1]. Die Notwendigkeit, geschlechtsspezifische Unterschiede in der Entwicklung von Suchterkrankungen zu untersuchen, wird offensichtlich, wenn man bedenkt, dass bereits auch in anderen Bereichen der Psychiatrie geschlechtsbezogene Unterschiede festgestellt wurden - so konnte gezeigt werden, dass hormonelle Veränderungen im Menstruations-, aber auch im weiblichen Lebenszyklus Einfluss auf die Psychobiologie psychiatrischer Erkrankungen ebenso wie auf die Pharmakokinetik bestimmter Medikamente zu haben scheinen [2].

Östrogenen dürfte dabei eine gewisse Rolle als „protektivem Faktor" zukommen, indem beispielsweise keine Veränderung des zerebralen Blutflusses bei kokainabhängigen Frauen in der östrogenen Zyklusphase festgestellt wurde, wohingegen männliche Kokainkonsumenten sowie Frauen in der gestagenen Zyklusphase verminderten zerebralen Blutfluss zehn Minuten nach intravenöser Kokainapplikation aufwiesen; daraus könnte die Vermutung abgeleitet werden, dass Östrogenen als biologischem Marker eine Mediatorrolle bei den durch Substanzabusus bedingten neurologischen Veränderungen zukommt. Der genaue Zusammenhang zwischen biologischen Grundlagen und ihrer Bedeutung für die Ausprägung von Suchtkrankheiten scheint jedoch noch weitgehend ungeklärt [3].

Geschlechtsbezogene Untersuchungen wurden bisher vorwiegend bei substanzabhängigen Schwangeren durchgeführt [1], denen sicherlich hinsichtlich der Auswirkungen des Suchtmittelkonsums auf das Neugeborene ein besonderer Status innerhalb des Patient(inn)enkollektivs Suchtkranker zukommt. Doch auch in diesem Bereich bedarf es noch intensiverer Forschung, um eine effizientere Therapie gewährleisten zu können.

Ziel dieser Arbeit ist es also, den Fokus auf die Notwendigkeit geschlechtsadäquater Behandlung in der Suchttherapie zu lenken - die Arbeit sollte einen Überblick über den aktuellen Stand der geschlechtsspezifischen Forschung bei Substanz- 
abhängigkeit geben und mögliche Ansätze in der Therapie derselben aufzeigen.

\section{Methode}

Es wurde in medizinischen Literaturverzeichnissen (MEDLINE, PUBMED) nach Publikationen in Bezug auf geschlechtsspezifische Unterschiede in der Suchttherapie gesucht. Die für die Autoren relevantesten Ergebnisse wurden zusammengefasst und hinsichtlich ihrer Bedeutung für die Forschung nach Prävalenz, Verlauf und Therapie der Suchtmittelabhängigkeit diskutiert.

\section{Ergebnisse}

Prävalenz

Obwohl fast dreimal so viele Männer davon berichten, illegale Substanzen zu konsumieren, und auch mehr Männer sich einer Therapie ihrer Substanzabhängigkeit unterziehen, zählt Drogenabhängigkeit zu der zweithäufigsten psychiatrischen Erkrankung, vor allem bei Frauen in der reproduktiven Phase [4]. Bei Frauen konnten unterschiedliche Risikofaktoren festgestellt werden, die zur Entwicklung einer Abhängigkeit von illegalen Substanzen, aber auch von legalen wie Alkohol oder Nikotin führen.

Während bei substanzabhängigen Patientinnen der Substanzmissbrauch häufig sowohl mit ,internalisierendem“ Verhalten wie Depression oder Suizidalität als auch mit „externalisierendem" Verhalten wie verminderter Impulskontrolle oder Aggressivität in Verbindung steht, konnte bei Männern nur eine Assoziation mit externalisierendem Verhalten nachgewiesen werden [5]. Zudem wurde in Studien bezüglich des Zusammenhangs zwischen PTSD (posttraumatic stress disorder) und SDSs (substance use disorders) ein auffällig hohes Vorkommen von PTSD bei substanzabhängigen Mädchen gefunden [6]. Wenn auch die Ursache für diesen Zusammenhang noch nicht ausreichend erklärt werden kann, könnte die Hypothese aufgestellt werden, dass bei Vorliegen ähnlicher Traumata Mädchen ein anderes Reaktionsmuster als Jungen aufweisen und insofern eine höhere Wahrscheinlichkeit für das Auftreten einer PTSD mit nachfolgendem Risiko eines Substanzmissbrauchs anzunehmen ist [7].

Bei der Untersuchung alkoholabhängiger Patient(inn)en konnte festgestellt werden, dass der familiäre Hintergrund (family history of alcoholism = FHA) einerseits sehr wohl einen Risikofaktor für die spätere Entwicklung von Alkoholabhängigkeit darstellt [8], andererseits häufig eine Relation zur FHV (family history of violence) aufweist, wobei vor allem bei Frauen ein direkter Zusammenhang zwischen FHA und CCP (childhood conduct problems) und Alkohol- bzw. Drogenkonsum im frühen Erwachsenenalter aufgezeigt wurde [9] - die Bedeutung der Konfrontation mit gewalttätigem Verhalten im Kindesalter dürfte allgemein auf die Entwicklung einer Substanzabhängigkeit bedeutenden Einfluss haben, nachdem bei betroffenen Frauen eine auffällig hohe Rate an physischem, psychischem und sexuellem Missbrauch zu verzeichnen ist. Insgesamt weisen substanzabhängige Frauen eine höhere Rate an psychiatrischer und auch an somatischer Komorbidität auf als männliche Patienten, was auf unterschiedliche Faktoren in der Genese des psychiatrischen
Krankheitsbildes schließen lässt. Auf psychologischer und sozialer Ebene konnte festgestellt werden, dass Frauen häufiger Beziehungsprobleme haben, Tendenz zu sozialer Isolation aufweisen und auch insgesamt eher Schwierigkeiten in der Sozialisierung zeigen. Im Gegensatz dazu sind substanzabhängige Männer wesentlich häufiger mit Rechtskonflikten konfrontiert, welche im Zusammenhang mit ihrer Krankheit auftreten [10].

Was die Entwicklung der Substanzmittelabhängigkeit betrifft, d.h. die Zeitspanne zwischen der erstmaligen Gelegenheit, illegale Substanzen zu konsumieren, und ihrem regelmäßigen Konsum bzw. Missbrauch, ergab eine Studie, dass Mädchen zwar früher in die Situation kommen, Drogen wie Marihuana, Kokain, Heroin und Halluzinogene zu konsumieren, was eventuell mit ihrer - im Vergleich zu Jungen - früher einhergehenden Reife in Verbindung gebracht werden könnte, andererseits Jungen prinzipiell mehr „Möglichkeiten“ haben, diverse Drogen zu konsumieren und diese auch eher wahrnehmen [11]. Für die Wahrscheinlichkeit der Entwicklung eines regelmäßigen Substanzkonsums nach einmaligem Gebrauch konnten zwar keine geschlechtsspezifischen Unterschiede nachgewiesen werden, jedoch scheint bei Frauen die Zeitspanne, aus einem Suchtmittelmissbrauch in eine Abhängigkeit zu gelangen, kürzer zu sein, als dies bei Männern der Fall ist [12]. Dies ist insofern von Bedeutung, als durch nähere Kenntnis der für die Entwicklung der Substanzabhängigkeit mitverantwortlichen Faktoren wesentliche Schritte im Bereich der Prävention unter Berücksichtigung geschlechtsspezifischer Aspekte unternommen werden können.

\section{Verlauf}

Auf somatischer Ebene dürfte bei Frauen eine höhere Vulnerabilität für die Konsequenzen des langfristigen Substanzabusus vorliegen - dies konnte unter anderem in Bezug auf Alkoholabhängigkeit sehr klar nachgewiesen werden: Hinsichtlich der bekannten toxischen Effekte von Alkohol wurde bei Frauen eine erhöhte Suszeptibilität für hepatische, kardiale, zerebrale und muskuläre Veränderungen beobachtet [13]. In einer weiteren Studie wurden die TDLE (total lifetime dose of ethanol) sowie andere Risikofaktoren für alkoholinduzierte Polyneuropathie berechnet und in Korrelation zur SEP (sensorisch evozierte Potenziale, abgeleitet am N. suralis) gesetzt: Im Vergleich zu männlichen Alkoholikern standen TDLE und Krankheitsdauer bei weiblichen Patientinnen in umgekehrter Proportionalität zur SEP-Amplitude; demnach tritt verminderte Sensitivität bei chronischen Alkoholikerinnen zu einem früheren Zeitpunkt auf, als dies bei alkoholkranken Männern der Fall ist, was auf eine erhöhte Empfänglichkeit peripherer Nervenfasern für die toxischen Effekte von Alkohol bei Frauen schließen lassen könnte [14]. Auf metabolischer Ebene könnten dem erhöhten körperlichen Fettanteil bei Frauen und dem bei gleicher Dosis Alkohol im Vergleich zu Männern höheren Blutspiegel Bedeutung für das unterschiedlich starke Auftreten alkoholbedingter Folgeerscheinungen zukommen [15] - die genauen Ursachen dafür, dass Frauen offensichtlich eine stärkere Prädisposition für alkoholinduzierte körperliche Schäden aufweisen, ist jedoch noch ungeklärt und bedarf weiterer Forschung. 
Auch beim Nikotinkonsum werden biologische Marker als Ursache für das erhöhte gesundheitliche Risiko von Frauen für tabakassoziierte Erkrankungen angenommen: Die Tatsache, dass die gleiche Anzahl konsumierter Zigaretten bei Frauen eher zu Atemwegserkrankungen führt, könnte ebenso durch ihre geschlechtsspezifisch kleinere Lungenoberfläche erklärt werden [16]. Hingegen gilt es als gesichert, dass Raucherinnen dem Risiko einer verminderten Fertilität, vorzeitiger Menopause, Osteoporose und weiterer hormonbedingter Erkrankungen ausgesetzt sind [17]. Die Assoziation zwischen Substanzmittelkonsum und geschlechtsadäquaten Folgeerscheinungen ist offensichtlich und lässt die Notwendigkeit der Entwicklung speziell auf Frauen abgestimmter Therapieschemata als nahe liegend erscheinen.

Bei substanzabhängigen Frauen konnte festgestellt werden, dass sie eher dazu neigen, begonnene Therapieversuche abzubrechen bzw. dass sie generell weniger dazu bereit sind, sich einer Therapie ihrer Substanzabhängigkeit zu unterziehen. Die Gründe dafür scheinen noch weitgehend unklar, jedoch gibt es Hinweise dafür, dass sowohl entsprechende Persönlichkeitsmerkmale als auch Ursachen, die im familiärsozialen Bereich liegen, dabei eine Rolle zu spielen scheinen [18]. So konnte in einer Studie bei kokainabhängigen Patient(inn)en dargelegt werden, dass vor allem bei Frauen mit aggressiven und misstrauischen Wesenszügen wesentlich öfter frühzeitige Therapieabbrüche zu verzeichnen sind, als dies bei Männern der Fall ist, die ein ebensolches Verhalten aufweisen [19]. Weitere Gründe, die für die schlechteren Behandlungsergebnisse bei substanzabhängigen Frauen mitverantwortlich sein könnten, sind Erziehungsverantwortung und die vorhandene Angst, dass die Bekennung zur Substanzabhängigkeit nach wie vor einer gewissen Stigmatisierung gleichkommt [20]. So ist es besonders bei graviden Substanzabhängigen sehr oft der Fall, dass sie sich erst relativ spät im Verlauf ihrer Schwangerschaft in medizinische Behandlung begeben, weil sie fürchten, dass öffentliche Einrichtungen ihnen die Kindesausfolgung verweigern könnten, was besonders bei dieser Patientinnengruppe ein zusätzliches Risiko für den Fötus bedeutet [21].

Weitere Untersuchungen sollten unternommen werden, um durch die Entwicklung spezifischer, auf die Bedürfnisse der betroffenen Patientinnengruppe abgestimmte Therapieprogramme, etwa die kontinuierliche Teilnahme substanzabhängiger Frauen an Erhaltungstherapien im Fall einer Opiatabhängigkeit, zu verstärken.

\section{Therapie}

Opioidabhängige Patient(inn)en bedürfen längerfristiger Therapie - die Illusion einer einmaligen Intervention ist ebenso obsolet und würde den aktuellen wissenschaftlichen Standards widersprechen [22] - und es gilt als gesichert, dass anhaltende Abstinenz zwar als erstrebenswertes Ziel erscheint, bei den meisten Patient(inn)en jedoch nicht als realisierbar anzusehen ist [23]. Vielmehr wird versucht, mit Hilfe der oralen Opioiderhaltungstherapie, bei der im Sinne der Diversifikation verschiedene Medikamente zur Auswahl stehen, eine psychosoziale Stabilisierung im Sinne einer Resozialisierung und Reintegration zu erreichen [25]. Prinzipiell sollte die Therapie substanzabhängiger Patient(inn)en aus einem multidisziplinären Programm bestehen, in dem neben der Rehabilitation durch ein psychosozial-medizinisches Therapieschema der Psychotherapie, insbesondere der Verhaltenstherapie, ein hoher Stellenwert beikommt [26]. Natürlich kann Psychotherapie keine Alternative zu medizinisch-therapeutischen Ansätzen bieten, jedoch stellt sie eine sinnvolle Ergänzung im Rahmen eines mehrstufigen Behandlungsspektrums dar: Nach erfolgreicher psychosozialer Stabilisierung und Einstellung auf ein synthetisches Opioid kann durch das Erlernen neuer Copingstrategien im Umgang mit „kritischen“ Situationen eine weitere Stabilisierung der Patient(inn)en gefördert werden. Es darf nicht vergessen werden, dass es sich bei Substanzabhängigkeit um eine schwere psychiatrische Krankheit handelt, die durch rezidivierende Rückfälle gekennzeichnet ist - umso wichtiger scheint es, den Patient(inn)en Hilfestellung beim Erlernen alternativer Verhaltensweisen anzubieten, wenn man bedenkt, dass Drogenabhängige markante Persönlichkeitsmerkmale wie geringe Frustrationstoleranz oder große Projektionsbereitschaft aufweisen [24].

Insbesondere bei substanzabhängigen Frauen hat sich ein interdisziplinäres Therapiesetting, bestehend aus medizinischer, psychosozialer und psychotherapeutischer Betreuung, als äußerst vorteilhaft erwiesen [25], wobei hier drogenabhängige Schwangere besonders hervorzuheben sind: In einer groß angelegten Studie an der Drogenambulanz des AKH Wien konnte nachgewiesen werden, dass im Rahmen eines multidisziplinären Betreuungsprogramms bestehend aus enger Zusammenarbeit zwischen Psychiater(inne)n, Sozialarbeiter(inne)n und einer Psychotherapeutin sowie Gynäko$\log$ (inn)en und Neonatolog(inn)en eine optimale Versorgung in der Betreuung der Patientinnen gewährleistet werden kann - dabei wurde u.a. festgestellt, dass die Chancen auf Obsorgerecht gravider Substanzabhängiger umso größer sind, je früher sich die Patientin in Opioiderhaltungstherapie begab [26]. Durch entsprechende Aufklärung könnte somit auch die Angst abhängiger Schwangerer vor öffentlichen Einrichtungen - viele Patient(inn)en fürchten, dass man ihnen die Kindesausfolgung verweigern könnte - vermindert werden [23]. Außerdem lassen sich durch eine möglichst frühe medizinische Intervention die durch Substanzkonsum potenziell auftretenden Komplikationen weitgehend vermindern bzw. vermeiden [27].

Multidisziplinäre Therapieansätze haben sich auch schon im Rahmen der Nikotinentwöhnung als effizient erwiesen: Nachdem Nikotin ein wesentlich höheres Suchtpotenzial aufweist als Heroin, gestaltet sich die Entwöhnung entsprechend schwierig und ist durch häufige Rückfälle gekennzeichnet. Wenn eine entsprechende Motivation der Patient(inn)en vorhanden ist, stellen verhaltenstherapeutische Maßnahmen in Kombination mit einer Nikotinersatztherapie in Form von Kaugummi, Transdermalpflaster oder Inhalator eine Chance zur erfolgreichen Raucherentwöhnung dar. Dem bei Frauen gefürchteten Problem einer Gewichtszunahme kann durch entsprechend niedrigkalorische Kost im Rahmen einer Ernährungsberatung entgegengewirkt werden. Speziell bei Raucherinnen scheint Verhaltenstherapie in Form von Gruppensitzungen sinnvoll, weil sie zum einen im Allgemeinen eine bessere Motivation zur Nikotinentwöhnung zeigen und zudem in der Therapie lernen, adäquate Bewältigungsstrategien im Umgang mit Stress und Konfliktsituationen zu entwickeln [28]. Untersuchungen haben gezeigt, dass Frauen eher in 
emotional negativ betonten Situationen rückfällig werden, während Männer häufiger im Zusammenhang mit sog. „social events“ ihre Abstinenz beenden [29].

Die angeführten Beispiele bezüglich der Berücksichtigung geschlechtsspezifischer Aspekte machen die Relevanz entsprechend adaptierter Therapieansätze zur Behandlung substanzabhängiger Patient(inn)en offensichtlich. Es bedarf jedoch weiterer Forschung, um diese Ansätze auszubauen mit der Aussicht, zukünftig noch effizientere Behandlungsstrategien gewährleisten zu können.

\section{Diskussion}

Die angeführten Beispiele bezüglich geschlechtsspezifischer Unterschiede bei Suchterkrankungen weisen keinen Anspruch auf Vollständigkeit auf, sondern sollen deutlich machen, dass zwar entsprechende Studien vorliegen und auch ansatzweise Therapieprogramme entwickelt wurden, die unterschiedliche geschlechtsspezifische Faktoren berücksichtigen - nach wie vor ist aber ein Mangel an entsprechenden Behandlungsprogrammen $\mathrm{zu}$ verzeichnen. Dies bedeutet, dass zukünftige Studien die Rolle des Geschlechts bei der Manifestation und Therapie von Suchterkrankungen berücksichtigen sollten. Die Wichtigkeit dieses Aspekts dürfte im Bereich der Suchtmedizin noch nicht ausreichend verstanden worden sein [10], wenn man bedenkt, dass bei anderen psychiatrischen Krankheiten wie der Schizophrenie bereits ein wesentlich breiteres Behandlungsspektrum etabliert wurde [24]. Wenn auch durch die Einführung von Langzeittherapieprogrammen Verbesserungen in der Behandlung substanzabhängiger Patient(inn)en erzielt werden konnten [30], ist es erstrebenswert, mit Hilfe intensiver Forschung auf dem Gebiet der Substanzabhängigkeit eine Erweiterung des medikamentösen Behandlungsangebots zu erzielen sowie durch die Etablierung psychotherapeutischer Maßnahmen zu komplementieren.

Sowohl bei Betrachtung der Nikotin- und Alkoholsucht als auch der Abhängigkeit von illegalen Substanzen unter wirtschaftlichem sowie unter medizinischem Aspekt scheint es plausibel, dass eine möglichst frühzeitig im Verlauf der Suchterkrankung einsetzende Behandlung wesentlicher zur Kostensenkung im Gesundheitssystem führen kann, als die Therapie der suchtassoziierten Folgeerscheinungen. Wiederum muss hier der Tatsache Rechnung getragen werden, dass die Prävalenz der Nikotinabhängigkeit, vor allem bei Frauen, noch immer bedenklich im Steigen ist. Ebenso erweisen sich palliative Maßnahmen wie Aufklärungskampagnen nur insofern als sinnvoll, wenn gleichzeitig auch die Möglichkeit entsprechender Behandlungssettings von Suchtkrankheiten gegeben ist.

Als wesentlicher Forschungsansatz gilt es zudem, die unterschiedliche geschlechtsspezifische Metabolisierung von Psychopharmaka zu untersuchen, da eventuell die schlechtere Langzeithaltequote bei Frauen durch zu geringe Medikamentenspiegel hervorgerufen sein dürfte. Um den Frauenanteil bei Untersuchungen zu erhöhen, sollten öffentliche Förderungen unter diesem Forschungsansatz vergeben werden.

\section{Literatur}

${ }^{1}$ Wheterington CL. Gender differences in the biological basis and progression to drug abuse: Implications for treatment. National Institute on Drug Abuse Research; Monograph series. Problems of Drug Dependence 2000: Proceedings of the 62nd Annual Scientific Meeting.

${ }^{2}$ Stein D, Nair M. Psychiatric aspects of women's health. International Clinical Psychopharmacology 2000; 15: S1-S4 (suppl 3)

${ }^{3}$ Kaufman MJ, Levin JM, Maas LC, Kukes TJ, Villafuerte RA, Dostal $\mathrm{K}$, Lukas SE, Mendelson JH, Cohen BM, Renshaw PF. Cocaineinduces cerebral vasoconstriction differs as a function of sex and menstrual cycle phase. Biol Psychiatr 49 2001; 9: 774-781

${ }^{4}$ Robins LN, Helzer JE; Weissmann MM, Orvaschel H, Burke JD, Regier DA. Lifetime prevalence of specific psychiatric disorders in the three sites. Arch Gen Psychiatry 1984; 41: 949-958

${ }^{5}$ Kulka RA, Schlenger WE, Fairbank JA, Hough RL, Lordan BK, Marmar CR, Weiss DA. Trauma and the Vietnam War generation: Report of findings from the National Vietnam Veterans Readjustment Study. New York: Bruner/Mazel, 1990

${ }^{6}$ Kessler RC, Sonnega A, Bromet E, Hughes M, Nelson CB. Posttraumatic stress disorder in the National Comorbidity Survey. Arch Gen Psychiatry 1995; 52: 1048-1060

${ }^{7}$ Breslau N, Davis GC, Andreski P, Peterson E. Traumatic events and posttraumatic stress disorder in an urban population of young adults. Arch Gen Psychiatry 1991; 48: 216-222

${ }^{8}$ Dawson DA, Harford TC, Grant BF. Family history as a predictor of alcohol dependence. Alcoholism Clin Exp Res 1992; 16: 572-575

${ }^{9}$ Chermack ST, Stoltenberg SF, Fuller BE, Blow FC. Gender differences in the development of substance-related problems: the impact of family history of alcoholism, family history of violence and childhood conduct problems. J Stud Alcohol 2000; 61: 845-852 (6)

${ }^{10}$ Jones HE. Gender differences in pharmacotherapy trials for opiate and cocaine dependent individuals. National Institute on Drug Abuse Research; Monograph series. Problems of Drug Dependence 2000: Proceedings of the 62nd Annual Scientific Meeting

${ }^{11}$ Van Etten ML, Anthony JC. Comparative epidemiology of initial drug opportunities and transitions to first use: marijuana, cocaine, hallucinogens and heroin. Drug Alcohol Depend 1999; 154: 117-125(2)

12 Anglin MD, Hser YI, McClothin W. Sex differences in addict careers. Am J Drug Alcohol Abuse 1987; 13: 59-71

${ }^{13}$ Urbano-Marquez A, Estruch R, Fernández-Sol J, Nicolás JM, Paré JC, Rubin E. Cardiomiopatia e miopatia alcolica: le donne sono più a rischio degli uomini. J Am Med Ass 1995; 274: 149-154

${ }^{14}$ Ammendola A, Gemini D, Iannaccone S, Argenzio F, Ciccone G, Ammendola E, Serio L, Ugolini G, Bravaccio F. Gender and peripheral neuropathy in chronic alcoholism: a clinical-electroneurographic study. Alcohol and Alcoholism 2000; 35: 368-371 (4)

${ }^{15}$ Frezza M, Di Padova C, Pozzato G. High blood alcohol levels in women: the role of decreased gastric alcohol dehydrogenase activity and firstpass metabolism. New England J Med 1990; 322: 95-99

${ }^{16}$ Langhammer A, Johnsen R, Holmen J, Gulsvik A, Bjermer L. Cigarette smoking gives more respiratory symptoms among women than among men. The Nord-Trondelag Health Study (Hunt). Epidemiol Community Health 2000; 54: 917-922 (12)

17 World Health Oranisation. Tobacco or health. Women and tobacco 1992: 31-65

18 Petry NM, Warren K. Gender differences in hostility of opioiddependent outpatients: role in early treatment termination. Drug Alc Depend 2000; 58: 27-33 
${ }^{19}$ Ball SA. The validity of an alternative five factor measure of personality in cocaine abusers 1995. Psychol Assess 1995; 7: $148-154$

${ }^{20}$ Woodhouse LD. An exploratory study of the use of life history methods to determine treatment needs for female substance abusers. Resp Victim Women Child 1990; 13: 12-15

${ }^{21}$ Deleon G, Jainchill N. Residential therapeutic communities for female substance abusers. Bull NY Acad Med 1991; 67: 277-290

22 Edwards G. Addiction treatment and the making of large claims. Addict 2000; 95: 1755-1757 (12)

${ }^{23}$ Hagan TA, Finnegan LP, Nelson-Zlupko L. Impedients to comprehensive treatment model for substance-dependent women: treatment and research questions. J psychoact drug 1994; 26 : 163-171

${ }^{24}$ Fischer G, Hauk R, Eder H, Pezawas L, Diamant K, Jagsch R, Pohl C. Das Betreuungsprofil im Wiener Allgemeinen Krankenhaus unter besonderer Berücksichtigung von Risikopopulationen. Neuropsychiatrie 1997; 4: 139-147

${ }^{25}$ Vogt I. Women and Drugs: A frame of reference for theory and practice. Women and Drugs. Straßburg: Council of Europe Press, 1993

${ }^{26}$ Ortner R, Schuster A, Rohrmeister C, Fischer G. Prädiktoren zur Stabilisierung opioidabhängiger gravider Frauen mit Ausblick auf das Obsorgerecht. Suchttherapie 2001; 2: 152-158

${ }^{27}$ Chasnoff IJ. Drugs, alcohol and the neonate: pay now or pay later. JAMA 1991; 266: 1567-1568

${ }^{28}$ Ortner R, Schindler SD, Kraigher D, Fischer G. Nikotinabhängigkeit bei Frauen; im Druck

${ }^{29}$ Fagerström KO, Tejding R, Westin A, Lunell E. Aiding reduction of smoking with nicotine replacement medications: hope for the recalcitrant smoker? Tob Control 1997; 6: 311-316 (4)

${ }^{30} \mathrm{O}$ 'Brien CP. Overview: The treatment of drug dependence. Addict 1994; 89: 1565-1569
Univ.-Prof. Dr. Gabriele Fischer

Drogenambulanz

Klinische Abteilung für Allgemeine Psychiatrie

Universitätsklinik für Psychiatrie

AKH Wien

Währinger Gürtel 18-20

1090 Wien

Österreich

E-mail: gabriele.fischer@akh-wien.ac.at 